\title{
Reemplazo de grano de sorgo con fruto de Guazuma ulmifolia en dietas de corderos con diferente forraje
}

\section{Replacement of sorghum grain with Guazuma ulmifolia fruit in lambs' diets with different forage}

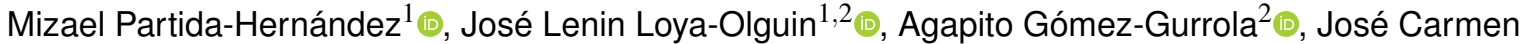

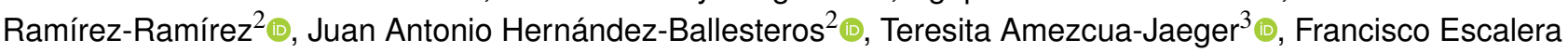 \\ Valente $^{1,2}$ (]), Leonor Sanginés-García ${ }^{3 *}$ (ㄷ) \\ ${ }^{1}$ Posgrado en Ciencias Biológico Agropecuarias. \\ ${ }^{2}$ Unidad Académica de Medicina Veterinaria y Zootecnia de la Universidad Autónoma de Nayarit. Carretera de Cuota \\ Compostela-Chapalilla Km 3.5, Compostela, Nayarit, México. \\ ${ }^{3}$ Departamento de Nutrición Animal, Instituto Nacional de Ciencias Médicas y Nutrición Salvador Zubirán. Vasco de Quiroga 15, \\ Delegación Tlalpan, Ciudad de México, México. \\ *Autor de correspondencia: leosangines@hotmail.com
}

Artículo científico recibido: 19 de agosto de 2017 aceptado: 07 de noviembre de 2018

RESUMEN. El objetivo del estudio fue evaluar el efecto de sustituir el grano de sorgo (SG) con fruto de Guazuma ulmifolia (GU) en dietas para corderos a base de alfalfa (AA) o maralfalfa (MA) sobre la digestibilidad y el comportamiento productivo. Se utilizaron cuatro ovinos machos Blackbelly de $35 \mathrm{~kg}$ de peso promedio, con cánula ruminal para determinar la digestibilidad in vivo, in vitro e in situ de la materia seca. En la prueba de comportamiento productivo se utilizaron 20 corderos Pelibuey de $15 \pm 1.5 \mathrm{~kg}$ de peso bajo un diseño completamente al azar. La composición porcentual de las dietas experimentales fue: dieta 1: 55 de MA, 20 de GU y 8 de SG; dieta 2: 55 de MA, 8 de GU y 20 de SG; dieta 3: 55 de AA, 20 de GU y 8 de SG y dieta 4: 55 de AA, 8 de GU y 20 de SG. Todas las dietas contenían melaza de caña de azúcar (5\%), pasta de canola $(9 \%)$ y mezcla de minerales (3\%). La sustitución de SG por GU en la dieta no afectó la digestibilidad de la materia seca ni el comportamiento productivo de los ovinos $(p>0.05)$. Pero los valores de digestibilidad y comportamiento productivo fueron inferiores $(p<0.05)$ con la inclusión de MA en las dietas. El grano de sorgo puede ser sustituido hasta en un $20 \%$ por el fruto de Guazuma ulmifolia en dietas de corderos, con niveles altos y bajos de fibra, sin afectar la digestibilidad de la materia seca ni el comportamiento productivo.

Palabras clave: Alimentos energéticos, árboles tropicales, borregos de pelo, digestibilidad, FDN.

ABSTRACT. The objective of this study was to evaluate the effect of replacement of sorghum grain (SG) with Guazuma ulmifolia fruit (GU) in lambs' diets with alfalfa (AA) or maralfalfa (MA) on productive performance and digestibility. Four male Blackbelly sheep with a mean body weight (BW) of $35 \mathrm{~kg}$, with ruminal cannula, were used for the determination of in vivo, in vitro and in situ digestibility. Twenty Pelibuey male lambs with a mean initial BW of $15 \pm 1.5 \mathrm{~kg}$ were used under a completely randomized design in the productive performance trial. The percentage composition of experimental diets were as follow: Diet 1: 55 of MA, 20 of GU and 8 of SG; Diet 2: 55 of MA, 8 of GU and 20 of SG; Diet 3: 55 of AA, 20 of GU and 8 of SG and Diet 4: 55 of AA, 8 of GU and 20 of SG. Moreover, all diets contain sugar cane molasses (5\%), canola meal $(9 \%)$ and mineral mix $(3 \%)$. There were no effect $(p>0.05)$ of sorghum grain replacement on digestibility and productive performance. Nevertheless, these value were lower $(p<0.05)$ with MA inclusion in diets. In conclusion, the sorghum grain can be replaced by Guazuma ulmifolia fruit up to $20 \%$ of the diets with low and high levels of fiber without effect on dry matter digestibility and productive performance.

Key words: Energetic feeds, digestibility, NDF, hair sheep, tropical trees. 


\section{INTRODUCCIÓN}

En regiones tropicales y subtropicales, la alimentación de pequeños rumiantes es a base de forraje (Kawas 2008). Pero la disponibilidad y calidad del mismo disminuyen en la época de baja o nula precipitación, además de que presentan alto contenido de carbohidratos estructurales y bajos valores de proteína, digestibilidad y energía metabolizable (Ku-Vera et al. 2013). Debido a la necesidad de suplementar con concentrados comerciales durante la época de seca, y por el aumento constante de los precios de los granos y alimentos proteicos, es factible considerar alimentos disponibles en la región (Hernández-Montiel et al. 2017), como los frutos de algunos árboles nativos, los cuales pueden mejorar el comportamiento productivo de los animales (Mlambo et al. 2007), debido a que tienen altos valores de proteína cruda (> $120 \mathrm{~g} \mathrm{~kg}^{-1}$ ) y digestibilidad in vitro $\left(540-800 \mathrm{~g} \mathrm{~kg}^{-1}\right.$ ) de la materia seca (Ku 2005).

La especie Guazuma ulmofilia es un árbol cuyas hojas sirven de forraje para el ganado (Olivares-Pérez et al. 2013), tiene frutos en forma de cápsulas verrugosas de 3 a $4 \mathrm{~cm}$ de largo (CATIE 1986) y presenta un contenido de proteína cruda entre 5.8 y 12.8\% (Pinto et al. 2004, Gómez et al. 2014). Por otra parte, el pasto tropical Maralfalfa (Pennisetum spp.) es una fuente importante de forraje para los animales por ser un pasto de corte de alto rendimiento y valor nutricional con contenidos de proteína cruda (PC) de hasta 16\% (Sosa et al. 2006). La alfalfa, por otro lado, es un forraje de excelente valor nutritivo con elevado contenido de proteína cruda, carbohidratos no estructurales (Xu et al. 2014), y digestibilidad superior a las gramíneas tropicales (Zabala 1995); pero su precio es mayor que el de los pastos. Por lo anterior, el objetivo del presente estudio fue evaluar el efecto de la sustitución parcial del grano de sorgo por fruto de Guazuma ulmifolia, utilizando alfalfa o maralfalfa como fuente de forraje en dietas para ovinos de pelo, sobre la digestibilidad y el comportamiento productivo.

\section{MATERIALES Y MÉTODOS}

El trabajo se realizó en la Unidad Académica de Medicina Veterinaria y Zootecnia (UAMVZ) de la Universidad Autónoma de Nayarit ubicada en Compostela, Nayarit, México ( $\left.21^{\circ} \mathrm{LN}, 104^{\circ} \mathrm{LO}\right)$ a $880 \mathrm{~m}$ de altitud, con clima semicálido-húmedo, temperatura media anual de $22^{\circ} \mathrm{C}$ y precipitación promedio de 1 000 mm (INEGI 2015).

El pasto maralfalfa se cosechó a los 90 días, se cortó a un tamaño de partícula de 2 a $4 \mathrm{~cm}$ y se secó al sol por $72 \mathrm{~h}$ con rotaciones cada $24 \mathrm{~h}$. El fruto maduro de Guazuma ulmifolia se colectó de árboles sin ningún tipo de manejo en Santa Isabel, municipio de Ahuacatlán, Nayarit, para luego trasladarlo a la UAMVZ donde se molió y secó a temperatura ambiente. La alfalfa se cosechó al inicio de la floración y se secó al sol durante $72 \mathrm{~h}$, para luego molerla en un molino de martillos con criba de una pulgada. Los otros ingredientes utilizados para elaborar las dietas experimentales se adquirieron en un establecimiento comercial. Se elaboraron cuatro dietas experimentales para cubrir las necesidades nutricionales de corderos destetados de acuerdo a las recomendaciones del NRC (1985), las cuales se diferenciaron por el tipo de forraje incluido (Pennisetum spp. o Medicago Sativa) y nivel de inclusión de fruto de Guazuma ulmifolia y grano de sorgo (Tabla 1).

Las dietas, la alfalfa, la maralfalfa y el fruto de Guazuma ulmifolia se analizaron (Tabla 2) siguiendo los métodos oficiales de la AOAC (2000): materia seca (método 930.15), proteína cruda (método 984.13), cenizas (método 942. 05) y extracto etéreo (método 991.36). La fibra detergente ácido (FDA) y fibra detergente neutro (FDN) se determinaron por el método descrito por Goering y Van Soest (1970) con un digestor (ANKOM ${ }^{\circledR}$ 200). También se determinó la digestibilidad in vitro (Tilley y Terry 1963) e in vivo (Schneider y Flatt 1975) de las dietas experimentales.

La determinación de degradabilidad ruminal in situ se realizó con la técnica de Ørskov et al. (1980), utilizando cuatro ovinos machos Blackbelly de $35 \mathrm{~kg}$ de peso vivo promedio, con cánulas ruminales. Los ovinos se alojaron en jaulas metabólicas individuales provistas de comederos y bebederos. La asignación 
Partida-Hernández et al.

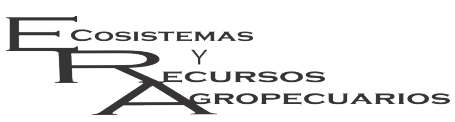

Fruto de guácima con diferentes forrajes

Ecosist. Recur. Agropec.

6(17):253-262,2019

Tabla 1. Ingredientes y composición química de dietas para corderos adicionadas con grano de sorgo y fruto de Guazuma ulmifolia, alfalfa o maralfalfa.

\begin{tabular}{lcccc}
\hline Ingrediente & Dieta 1 & Dieta 2 & Dieta 3 & Dieta 4 \\
\hline Maralfalfa & 55.0 & 55.0 & 0 & 0 \\
Alfalfa & 0 & 0 & 55.0 & 55.0 \\
Guazuma ulmifolia & 20.0 & 8.0 & 20.0 & 8.0 \\
Sorgo & 8.0 & 20.0 & 8.0 & 20.0 \\
Canola & 9.0 & 9.0 & 9.0 & 9.0 \\
Melaza & 5.0 & 5.0 & 5.0 & 5.0 \\
Mezcla de minerales & 3.0 & 3.0 & 3.0 & 3.0 \\
Composición química Materia seca & $88.0 \pm 0.43$ & $87.5 \pm 0.3$ & $83.6 \pm 0.3$ & $85.3 \pm 0.3$ \\
Proteína cruda & $13.3 \pm 0.3$ & $12.6 \pm 0.6$ & $18.0 \pm 0.2$ & $16.3 \pm 0.1$ \\
Cenizas & $6.6 \pm 0.3$ & $6.3 \pm 0.0$ & $6.9 \pm 0.1$ & $6.6 \pm 0.1$ \\
Fibra detergente neutra & $55.3 \pm 0.4$ & $50.0 \pm 0.8$ & $40.7 \pm 1.1$ & $35.4 \pm 0.3$ \\
Fibra detergente ácida & $32.6 \pm 0.0$ & $29.1 \pm 1.0$ & $24.8 \pm 0.5$ & $21.3 \pm 0.5$ \\
Extracto etéreo & $3.7 \pm 0.2$ & $3.5 \pm 0.5$ & $3.0 \pm 0.1$ & $2.9 \pm 0.2$ \\
TND & 57.6 & 59.1 & 59.1 & 60.8 \\
Energía metabolizable (Mcal $\mathrm{kg}^{-1}$ ) & 2.5 & 2.6 & 2.6 & 2.7 \\
\hline
\end{tabular}

Tabla 2. Composición química de los ingredientes de dietas para corderos adicionadas con grano de sorgo y fruto de Guazuma ulmifolia, alfalfa o maralfalfa.

\begin{tabular}{lrrrr}
\hline & Alfalfa & Maralfalfa & Guazuma ulmifolia & Sorgo (NRC) \\
\hline Materia seca & $89.2 \pm 0.1$ & $87.9 \pm 0.3$ & $93.0 \pm 0.4$ & 89.0 \\
Proteína cruda & $23.6 \pm 0.0$ & $11.3 \pm 0.7$ & $14.5 \pm 1.1$ & 11.0 \\
Cenizas & $9.6 \pm 0.2$ & $9.1 \pm 1.1$ & $4.4 \pm 0.1$ & 2.0 \\
Extracto etéreo & $2.0 \pm 0.7$ & $2.8 \pm 0.1$ & $4.6 \pm 0.3$ & 3.0 \\
FDN & $49.3 \pm 0.5$ & $73.0 \pm 2.1$ & $59.2 \pm 0.7$ & 15.0 \\
FDA & $44.8 \pm 1.2$ & $42.9 \pm 1.9$ & $35.1 \pm 1.8$ & 6.0 \\
\hline
\end{tabular}

de las dietas experimentales se realizó bajo un diseño cuadrado latino $4 \times 4$. Cada periodo tuvo una duración de 21 días. Los primeros 14 días de cada periodo, fueron de adaptación de los animales a la dieta y del 15 al 18 se prepararon las bolsas de nyIon $(12 \times 8 \mathrm{~cm}$ con porosidad de 1200 a 1600 orificios por $\mathrm{cm}^{2}$ ) con muestras de las dietas experimentales que se incubaron en el rumen durante $0,3,6,9,24$, $30,36,48$ y $60 \mathrm{~h}$ para determinar la degradabilidad in situ. Después de la incubación las bolsas de todos los tiempos se lavaron cinco veces por un minuto hasta obtener un líquido del enjuague claro. Después del lavado las bolsas se secaron a $65^{\circ} \mathrm{C}$ por $48 \mathrm{~h}$, enseguida se calculó la desaparición de la materia seca en el rumen con el programa NEWWAY aplicando el modelo exponencial $\mathrm{Y}=\mathrm{a}+\mathrm{b}\left(1-\mathrm{e}^{-c t}\right)$, donde $\mathrm{Y}$ es la degradación de la materia seca en el tiempo t, a es la fracción soluble, b es la fracción insoluble potencialmente degradable, c es la tasa de degradación y t es el tiempo (Ørskov et al. 1980).

Del día 19 al 21 de cada periodo, antes del suministro de alimento, se realizó la recolección to- tal de heces de cada animal colocando una bolsa con arnés. Se tomó una muestra del $10 \%$ del total de heces de cada animal y se almacenó en bolsas individuales estériles a $-20^{\circ} \mathrm{C}$ hasta su análisis. Se formó una muestra compuesta de las heces de cada animal y se le determinó el porcentaje de materia seca en una estufa de aire forzado a $65^{\circ} \mathrm{C}$ por $72 \mathrm{~h}$ para la determinación digestibilidad in vivo (Schneider y Flatt 1975).

Para la prueba de comportamiento productivo se utilizaron 20 ovinos machos Pelibuey de $10.6 \pm$ $1.6 \mathrm{~kg}$ de peso vivo, los cuales fueron tratados con ivermectina al $1 \%$ para el control de parásitos internos y externos. Los ovinos se alojaron en corrales individuales con comedero y bebedero automático. A cada cordero se le asignó, al azar, una de las cuatro dietas experimentales con cinco repeticiones por tratamiento, la cual recibió, durante 100 días. Los animales tuvieron un periodo de adaptación a las dietas de 15 días, durante los cuales se incorporó la dieta experimental de manera gradual, incrementando $20 \%$ de la dieta cada tres días. Luego del pe- 
riodo de adaptación, los animales se pesaron, antes de ser alimentados, para obtener el peso inicial. El alimento se ofreció todos los días a las $08: 00 \mathrm{~h}$, después de retirar y pesar el alimento rechazado del día anterior. Cada día se ofreció el 5\% más del consumido el día anterior con la finalidad de garantizar el consumo a libre acceso. Los animales se pesaron al final del experimento, previo a la administración del alimento.

Se utilizó un diseño cuadrado latino 4 x 4 para el análisis de los datos obtenidos en las pruebas de degradabilidad ruminal y digestibilidad in vivo. Los resultados de la digestibilidad in vitro y el comportamiento productivo se analizaron con análisis de varianza bajo un diseño completamente al azar con el paquete estadístico de SAS (SAS 2004). La diferencia entre medias se determinó con la prueba de Tukey al 5\% (Herrera y Barrera 2005).

\section{RESULTADOS}

En la Tabla 2 se presenta la composición química del fruto de Guazuma ulmifolia, alfalfa y maralfalfa. El porcentaje de proteína cruda (PC) de la maralfalfa ( $11 \%$ ) fue inferior a lo esperado, mientras que el contenido de PC del fruto de G. ulmifolia (14\%) fue superior que el del pasto maralfalfa. Además, el contenido de PC del grano de sorgo fue menor al compararlo con el fruto de G. ulmifolia. El contenido de fibra detergente neutra (FDN) del fruto de G. ulmifolia fue menor que el del pasto maralfalfa, mientras que la alfalfa presentó el valor más bajo. Los contenidos de FDN y FDA del fruto de G. ulmifolia fueron 4 y 6 veces más altos, respectivamente, comparados con los del grano de sorgo.

El análisis químico mostró que las dietas adicionadas con alfalfa tuvieron mayor porcentaje de proteína cruda y menor contenido de FDN y FDA. Por otro lado, los porcentajes de las fracciones de fibra (FDN y FDA) de las dietas con $20 \%$ de fruto de $G$. ulmifolia fueron superiores que a aquellas con $20 \%$ de grano de sorgo. Todas las dietas cubrieron el requerimiento de energía metabolizable (1.4 Mcal día $^{-1}$ ) de corderos de $10 \mathrm{~kg}$ de peso vivo con crecimiento moderado y $200 \mathrm{~g} \mathrm{día}^{-1}$ de ganancia diaria (Tabla 1) del NRC. Las dietas con alfalfa presentaron valores mayores $(p<0.05)$ de digestibilidad de la materia seca in vivo (DMSIV) e in vitro (DMSIVT) que aquellas con maralfalfa, sin encontrar diferencia $(p<0.05)$ entre las dietas con diferentes niveles de grano de sorgo (Tabla 3). La DMSIVT de la dieta con $55 \%$ de maralfalfa y $20 \%$ de fruto de $G$. ulmifolia fue menor $(p<0.05)$ que la dieta con la misma cantidad de maralfalfa y $20 \%$ de sorgo, pero la DMSIV no mostró diferencia significativa $(p>0.05)$ entre ellas.

Las diferencias entre los valores de desaparición in situ de la materia seca de las dietas experimentales (Tabla 4), coinciden con aquellas observadas de digestibilidad in vivo e in vitro. Sin embargo, los valores varían con la técnica empleada. El potencial de degradación y las fracciones insolubles potencialmente digestible fueron mayores $(p<0.05)$ en las dietas con alfalfa respecto a las dietas con maralfalfa, pero no se observó diferencia $(p>0.05)$ entre las dietas con diferentes niveles de grano de sorgo cuando se combinaron con alfalfa o maralfalfa. El consumo de materia seca, peso final y ganancia de peso fueron menores $(p<0.05)$ en los animales que consumieron las dietas con maralfalfa.

\section{DISCUSIÓN}

El contenido de proteína cruda del fruto de G. ulmifolia es superior a lo reportado en otros trabajos (Pinto et al. 2004). Los porcentajes de las fracciones de fibra se encuentran dentro de los rangos reportados por Gómez et al. (2014) y Rojas et al. (2015), con valores entre 44 y $64 \%$ de FDN y de 34 a $54 \%$ de FDA. Mientras que el contenido de FDN fue similar a lo reportado por Román et al. (2008). La variación en la composición química del fruto de G. ulmifolia, reportada puede deberse a las condiciones edafoclimáticas del sitio de producción, época del año, madurez, lugar de colecta, variedad y manejo del árbol (López et al. 2008, Zardo y Henriquez 2011). La semejanza de los contenidos de PC y fracciones de fibra del fruto de guácima obtenidos en el presente estudio y los reportados por Gómez et al. (2014) pueden explicarse por el mismo sitio de 
Partida-Hernández et al.

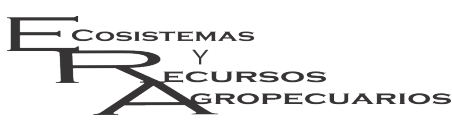

Fruto de guácima con diferentes forrajes

Ecosist. Recur. Agropec.

6(17):253-262,2019

Tabla 3. Digestibilidad in vitro e in vivo de la materia seca de dietas para corderos adicionadas con grano de sorgo y fruto de Guazuma ulmifolia, alfalfa o maralfalfa.

\begin{tabular}{|c|c|c|c|c|c|c|}
\hline & Dieta 1 & Dieta 2 & Dieta 3 & Dieta 4 & EEM & $\mathrm{P}$ \\
\hline in vitro & $42.5 \pm 0.5^{c}$ & $49.1 \pm 2.7^{b}$ & $66.9 \pm 1.6^{a}$ & $69.1 \pm 2.6^{a}$ & 2.0 & $<.0001$ \\
\hline in vivo & $53.0 \pm 3.9^{b}$ & $51.2 \pm 5.2^{b}$ & $69.3 \pm 3.4^{a}$ & $71.64 \pm 6.1^{a}$ & 4.9 & 0.0021 \\
\hline
\end{tabular}

Tabla 4. Desaparición in situ de la materia seca de dietas para corderos adicionadas con grano de sorgo y fruto de Guazuma ulmifolia, alfalfa o maralfalfa.

\begin{tabular}{|c|c|c|c|c|c|c|c|}
\hline & Tasa de Pasaje & Dieta 1 & Dieta 2 & Dieta 3 & Dieta 4 & EEM & $P$ \\
\hline (a) Fracción soluble & & $4.57 \pm 2.81^{a}$ & $7.12 \pm 4.64^{a}$ & $6.47 \pm 1.25^{a}$ & $7.62 \pm 4.81^{a}$ & 3.85 & 0.70 \\
\hline $\begin{array}{l}\text { (b) Fracción insoluble } \\
\text { potencialmente degradable }\end{array}$ & & $31.15 \pm 4.06^{b}$ & $38.75 \pm 5.90^{b}$ & $57.4 \pm 9.79^{a}$ & $63.8 \pm 7.91^{a}$ & 5.55 & $<0.01$ \\
\hline (c) Tasa de pasaje & & $0.067 \pm 0.02^{a}$ & $0.04 \pm 0.02^{a}$ & $0.069 \pm 0.02^{a}$ & $0.064 \pm 0.02^{a}$ & 0.03 & 0.61 \\
\hline Hora cero & & $4.41 \pm 0.89^{a}$ & $4.35 \pm 0.58^{a}$ & $3.30 \pm 0.11^{a}$ & $4.03 \pm 0.45^{a}$ & 0.51 & 0.73 \\
\hline $\begin{array}{l}\text { Degradabilidad de fracción } \\
\text { insoluble en agua }\end{array}$ & & $31.33 \pm 4.56^{b}$ & $41.52 \pm 8.92^{b}$ & $62.45 \pm 7.14^{a}$ & $64.50 \pm 10.07^{a}$ & 6.37 & $<0.01$ \\
\hline Potencial de degradabilidad & & $35.75 \pm 4.59^{c}$ & $45.9 \pm 8.94 b^{c}$ & $63.87 \pm 9.18^{a}$ & $71.37 \pm 10.11^{a}$ & 6.38 & $<0.01$ \\
\hline & 0.02 & $28.07 \pm 2.24^{b}$ & $30.35 \pm 3.65^{b}$ & $51.9 \pm 5.79^{a}$ & $51.42 \pm 4.41^{a}$ & 3.03 & $<0.01$ \\
\hline Degradabilidad efectiva & 0.05 & $21.95 \pm 1.19^{b}$ & $22.57 \pm 3.63^{b}$ & $40.55 \pm 6.36^{a}$ & $38.8 \pm 2.85^{a}$ & 3.46 & $<0.01$ \\
\hline & 0.08 & $18.37 \pm 0.76^{b}$ & $18.9 \pm 3.06^{b}$ & $33.97 \pm 6.73^{a}$ & $31.72 \pm 1.97^{a}$ & 3.77 & $<0.01$ \\
\hline
\end{tabular}

$a, b, c$ literales distintas en la misma fila indican diferencia estadísticamente $(\mathrm{p}<0.05), \pm$ Desviación Estándar, Tasa de pasaje expresada en \% $\mathrm{h}^{-1}$.

cosecha, aunque se realizaron en diferente año.

De acuerdo con Di Marco (2011), la calidad de un forraje es alta cuando contiene menos del $50 \%$ de FDN y más del $15 \%$ de PC, mientras que uno de baja calidad, tiene más del $65 \%$ de FDN y menos del $8 \%$ de PC. Por lo que la alfalfa puede considerarse como un forraje de alta calidad, mientras que la maralfalfa de mediana a baja calidad lo cual probablemente influyó en los resultados de la digestibilidad de las dietas y el comportamiento productivo de los corderos.

El contenido de FDN se relaciona de forma negativa con la digestibilidad de los alimentos. En ese sentido, Na et al. (2017) reportaron que al aumentar la cantidad de forraje en las dietas se disminuye la digestibilidad de la materia seca y orgánica en rumiantes. Debido a que se incrementa el porcentaje de FDN de la dieta, misma que se compone de carbohidratos estructurales, los cuales son de baja o lenta digestibilidad. En el presente trabajo, el contenido de FDN de los forrajes influyó en el contenido de este componente en las dietas y en la digestibilidad, aunque tuvieran las mismas proporciones de forraje:concentrado. Probablemente, la alfalfa podría ser sustituida por un pasto, como la maralfalfa, si las dietas tuvieran contenidos similares de FDN (Eastridge et al. 2009).

Los valores de digestibilidad de la materia seca, in vitro e in vivo (Tabla 3 ), de las dietas con alfalfa fueron mayores $(p<0.05)$ que aquellas con maralfalfa, mientras que la digestibilidad in vitro de la dieta con maralfalfa y $8 \%$ de sorgo fue menor ( $p$ $<0.05)$ que aquella con el mismo forraje y $20 \%$ de grano de sorgo. Debido a que el mayor contenido de FDN y FDA de la G. ulmifolia respecto al sorgo (Tabla 2) ocasionó que las dietas con niveles más elevados de G. ulmifolia tuvieran mayores contenidos de las fracciones de fibra. La digestibilidad in vitro de la dieta con alfalfa y $G$. ulmifolia es semejante a la reportada con dietas con 15 y $30 \%$ de fruto de $G$. ulmifolia con 29 y $12 \%$, respectivamente, de mazorca de maíz con $21.8 \%$ de FDN (Rojas et al. 2015). La estructura, la cantidad y tipo de carbohidratos almacenados en el forraje determinan la digestibilidad o disponibilidad para las bacterias ruminales (Hall et al. 1998). Además, los porcentajes de proteína cruda, carbohidratos no fibrosos, fibra detergente neutro y la digestibilidad de la FDN son diferentes entre los forrajes (Xu et al. 2014). Por lo que la diferencia en la digestibilidad entre tratamientos del presente experi- 
mento, se puede atribuir al contenido de FDN y PC de los forrajes y los ingredientes energéticos como el grano de sorgo y el fruto de G. ulmifolia. Por otra parte, los valores diferentes de digestibilidad entre experimentos pueden ser explicados por la proporción forraje:concentrado y la combinación de ingredientes de las dietas.

La fracción insoluble potencialmente degradable y la degradabilidad efectiva fueron mayores $(p<0.05)$ en las dietas con alfalfa que aquellas con maralfalfa. La eficiencia para el aprovechamiento de los alimentos por parte de los rumiantes depende de dos aspectos críticos en el proceso de fermentación: 1) velocidad de fermentación o tasa de degradación, y 2) velocidad de paso o tasa de pasaje (Van Soest 1994, Fox et al. 2000). La degradación más lenta de la dieta con $55 \%$ de maralfalfa y $8 \%$ de fruto de G. ulmifolia comparada con la dieta con el mismo porcentaje de maralfalfa y $20 \%$ de G. ulmifolia favoreció el incremento de la fracción potencialmente digestible, el potencial de degradación y la degradabilidad efectiva en rumen; lo que es congruente con los resultados de digestibilidad in vitro e in vivo. Sin embargo, los valores degradabilidad in situ no son iguales a los de digestibilidad in vivo e in vitro, aunque estos últimos dos se asemejen. Esto se debe a que los valores de digestibilidad in vitro e in vivo estiman la digestibilidad en todo el tracto digestivo, mientras que la digestibilidad in situ solo en el rumen.

Los resultados de comportamiento productivo (Tabla 5) coinciden con los de degradabilidad y digestibilidad, ya que el consumo de materia seca, peso final y ganancia de peso fueron menores $(p<$ 0.05 ) en los animales que consumieron las dietas con maralfalfa. Al respecto Oba y Allen (1999) mencionan que la digestibilidad in vitro e in situ se relacionan con el consumo de materia seca y el contenido de FDN. En este sentido, Allen (2000) observó que al utilizar dietas para ovinos con más de $25 \%$ de FDN, disminuyó el consumo, lo que influye en la ganancia de peso de los animales. Al respecto Lu et al. (2008) observaron una disminución en el consumo de materia seca en cabras que recibieron alimento con más del $41 \%$ de FDN, lo que indica que alimentar con dietas altas en fibra, disminuyen la ganancia de peso, debido a que el consumo de forraje está asociado al llenado del rumen y la cantidad de materia seca ingerida (Doyle et al. 1988). Todas las dietas rebasaron los niveles de FDN recomendados; pero las dietas con maralfalfa tenían más del $35 \%$ de FDN que las que contenían alfalfa, lo que ocasionó el aumento en el consumo de materia seca y la ganancia de peso.

La cantidad y composición de fibra presente en la dieta es un factor determinante en el consumo y digestibilidad de la misma, ya que hay correlación negativa entre FDA y la digestibilidad (Piñeiro 2013). En ese sentido, la calidad del forraje tuvo efecto directo en los parámetros productivos, ya que los animales que consumieron dietas con alfalfa presentaron mejores resultados en las variables estudiadas, sin mostrar efecto la sustitución parcial del grano de sorgo por el fruto de G. ulmifolia en la dieta (Tabla 5). A pesar de que las dietas con mayor porcentaje de fruto de G. ulmifolia tuvieron mayor contenido de proteína, el consumo de materia seca y las ganancias diarias de peso no fueron afectadas $(p>0.05)$. El incremento de proteína en las dietas puede mejorar el consumo cuando los animales consumen forraje de baja calidad (Salisbury et al. 2004), pero el consumo de materia seca no se modifica si rebasa el nivel máximo de consumo de FDN de $12.5 \mathrm{~g} \mathrm{~kg}^{-1}$ de peso (Bohnert et al. 2002), lo que fue rebasado por todos los borregos del presente experimento.

Las ganancias diarias de peso (GDP) son similares a las obtenidas por Díaz et al. (1995) y Lu et al. (2008) al alimentar cabras con niveles semejantes de FDN (Tabla 5). Por otra parte, los borregos alimentados con maralfalfa tuvieron ganancias similares a las de ovinos Pelibuey alimentados con pastos tropicales como Taiwan (González et al. 2011), mientras que las GDP de los borregos que recibieron alfalfa coinciden con lo reportado por Peralta et al. (2004) quienes incluyeron 10 y $20 \%$ de harina de parota en sustitución del grano, con 80:20 concentrado:forraje.

Los resultados con frutos arbóreos en la alimentación de ovinos y caprinos es variada, debido a la composición y la relación de forraje:concentrado de las dietas. Estudios con fruto de parota (Enterolobium cyclocarpum), como sustituto de grano de sorgo 
Tabla 5. Comportamiento productivo de corderos con dietas con diferentes niveles de grano de sorgo y fruto de Guazuma ulmifolia combinadas con alfalfa o maralfalfa.

\begin{tabular}{lccccr}
\hline Variable & Dieta 1 & Dieta 2 & Dieta 3 & Dieta 4 & $\mathrm{P}$ \\
\hline PV Inicial (kg) & $10.6 \pm 1.6^{b}$ & $10.1 \pm 1.4^{b}$ & $12.6 \pm 1.8^{a}$ & $12.3 \pm 1.1^{a}$ & 0.0400 \\
PV Final (kg) & $15.1 \pm 1.3^{b}$ & $16.0 \pm 1.1^{b}$ & $30.3 \pm 4.0^{a}$ & $32.8 \pm 2.5^{a}$ & 0.0001 \\
Ganancia de peso Total (kg) & $4.5 \pm 0.9^{b}$ & $5.9 \pm 1.2^{b}$ & $17.7 \pm 3.0^{a}$ & $20.5 \pm 2.1^{a}$ & 0.0001 \\
GDP $\left(\mathrm{g} \mathrm{d}^{-1}\right)$ & $41 \pm 8.4^{b}$ & $54 \pm 1.5^{b}$ & $162 \pm 27.7^{a}$ & $187 \pm 18.8^{a}$ & 0.0001 \\
CMS $\left(\mathrm{g} \mathrm{d}^{-1}\right)$ & $562 \pm 55.8^{b}$ & $626 \pm 7^{b}$ & $1232 \pm 139.7^{a}$ & $1254 \pm 59.8^{a}$ & 0.0001 \\
Conversión alimenticia & $14.2 \pm 3.9^{a}$ & $12.1 \pm 3.5^{a b}$ & $7.7 \pm 0.8^{b c}$ & $6.7 \pm 0.7^{c}$ & 0.0009 \\
\hline a,b,c literales en la misma fila indican diferencia estadísticamente $(\mathrm{p}<0.05) . \pm$ Desviación Estándar. PV: peso \\
vivo, GDP: ganancia diaria de peso, CMS: consumo de materia seca.
\end{tabular}

y pasta de algodón, se obtuvieron ganancias diarias de peso de $229 \mathrm{~g}$ en borregos Katahdin, Blackbelly y sus cruzas (Moscoso et al. 1995). Valores que son superiores a los encontrados en el presente estudio, lo que se puede deber a que los animales tuvieron un peso inicial mayor $(18 \mathrm{~kg})$, mientras que Álvarez et al. (2003) observaron una ganancia diaria de peso (86.74 $\mathrm{g} \mathrm{día}^{-1}$ ), consumo de alimento inferiores (460 g día ${ }^{-1}$ ) y conversión alimenticia similar (7.47) al adicionar $30 \%$ de fruto de parota en la dieta.

\section{CONCLUSIONES}

Es importante para los productores contar con alimentos energéticos, como el fruto de G. ulmifolia, que tiene mayor cantidad de proteína cruda que los cereales pero un menor costo, aunque su contenido de fracciones de fibra es alto. El grano de sorgo puede sustituirse de forma parcial por el fruto de $G$. ulmifolia en dietas de corderos que consumen forraje de alta y baja calidad, sin afectar la digestibilidad o el comportamiento productivo de los animales.

\section{LITERATURA CITADA}

Allen MS (2000) Effects of diet on short-term regulation of feed intake by lactating dairy cattle. Journal of Dairy Science 83: 1598-1624.

AOAC (2000) Association of Official Analytical Chemists. Official Methods of Analysis. 17th edition. Gaithersburg, MD, USA. 141p.

Álvarez-Morales G, Melgarejo-Velázquez L, Castañeda-Nieto Y (2003) Ganancia de peso, conversión y eficiencia alimentaria en ovinos alimentados con fruto (semilla con vaina) de parota (Enterolobiumcyclocarpum) y pollinaza. Veterinaria México 34: 39-46.

Bohnert DW, Schauer CS, Bauer ML, DelCurto T (2002) Influence of rumen protein degradability and supplementation frequency on steers consuming low-quality forage: I. Site of digestion and microbial efficiency. Journal of Animal Science 80: 2967-2977.

CATIE (1986) Silvicultura de especies promisorias para la producción de leña en América Central. Centro Agronómico Tropical de Investigación y Enseñanza. Turrialba, Costa Rica. 228p.

Di Marco O (2011) Estimación de calidad de los forrajes. Revista Producir XXI: 24-30.

Díaz Y, Escobar A, Viera J (1995) Efecto de la substitución parcial del suplemento convencional por follaje de pachecoa (Pachecoa venezuelensis) o gliricidia (Gliricidia sepium) en la alimentación de corderos postdestete. Livestock Research for Rural Development 7: 1.

Doyle PT, Dove H, Freer M, Hart FJ, Dixon RM, Egan AR (1988) Effects of a concentrate supplement on the intake and digestion of a low-quality forage by lambs The Journal of Agricultural Science 111: 503-511. 
Eastridge ML, Bucci PB, Ribeiro CVDM (2009) Feeding equivalent concentrations of forage neutral detergent fiber from alfalfa hay, grass hay, wheat straw, and whole cottonseed in corn silage based diets to lactating cows. Animal Feed Science and Technology 150: 86-94.

Fox DG, Tylutki TP, Tedeschi LO, Van Amburgh ME, Chase LE, Pell AN, et al. (2000) The net carbohydrate and protein system for evaluating herd nutrition and nutrient excretion: CNCPS version, 5(0), M1. Cornell University. Ithaca, NY, USA. 292p.

Goering HK, Van Soest PJ (1970) Forage fiber analyses (apparatus, reagents, procedures and some applications). USDA. Washington DC, USA. 20p.

Gómez GA, Partida HM, Ramírez DR, Ramírez RJC, Gómez GJA, González MM, et al. (2014) Efecto de la inclusión del fruto de Guazuma ulmifolia como sustituto del maíz en la dieta sobre el comportamiento productivo y rendimiento en canal de ovinos pelibuey. Tropical and Subtropical Agroecosystems 17: 215222.

González-Garduño R, Torres-Hernández G, Arece-García J (2011) Ganancia de peso de ovinos alimentados con pasto Taiwán (Pennisetum purpureum) suplementados con diversas fuentes de proteína. Avances en Investigación Agropecuaria 15: 3-20.

Hall MB, Pell AN, Chase LE (1998) Characteristics of neutral detergent soluble fiber fermentation by mixed ruminal microbes. Animal Feed Science and Technology 70: 23-39.

Hernández-Montiel W, Ramos-Juárez, JA, Aranda-lbáñez EM, Hernández-Mendo O, Munguía-Flores VM, \& Oliva-Hernández J (2017). Uso potencial y limitantes de la leguminosa Canavalia ensiformis en la salud y productividad de los ovinos. Ecosistemas y recursos agropecuarios, 4: 187-200.

Herrera HJG, Barrera SA (2005) Análisis estadístico de experimentos pecuarios. Manual de experimentos pecuarios. Manual de Procedimientos (Aplicaciones del Programa SAS). Colegio de Postgraduados. México. $119 p$

INEGI (2015) Anuario estadístico del Estado de Nayarit. Instituto Nacional de Estadística y Geografía. Gobierno del Estado de Nayarit. Aguascalientes, Aguascalientes, México. 450p.

Kawas JR (2008) Producción y utilización de bloques multinutrientes como complemento de forrajes de baja calidad para caprinos y ovinos: la experiencia en regiones semiáridas. Tecnología y Ciencia Agropecuaria 2: 63-69.

Ku J (2005) Nutritive value of trees and shrubs for ruminants. In: Mosquera-Losada MR, Rigueiro-Rodríguez A, McAdam R (eds) Silvopastoralism and sustainable land management. CAB International. Wallingford, UK. pp: 83-86.

Ku-Vera JC, Ayala-Burgos AJ, Solorio-Sánchez FJ, Briceño-Poot EG, Ruiz-González A, Piñeiro-Vázquez AT, et al. (2013) Tropical tree foliages and shrubs as feed additives in ruminant rations. In: Nutritional Strategies of Animal Feed Additives. Nova Sci. Publishers. USA. pp: 59-76.

López HMA, Rivera LJA, Ortega RL, Escobedo MJG, Magaña MMA, Sanginés GJR, et al. (2008) Contenido nutritivo y factores antinutricionales de plantas nativas forrajeras del norte de Quintana Roo. Técnica Pecuaria en México 46: 205-215.

Lu CD, Kawas JR, Mahgoub OG (2008) Recent advancements in fiber digestion and utilization in goats. Tropical and Subtropical Agroecosystems 9: 65-72. 
Mlambo V, Sikosana JLN, Mould FL, Smith T, Owen E, Mueller-Harvey I (2007) The effectiveness of adapted rumen fluid versus PEG to ferment tannin-containing substrates in vitro. Animal Feed Science and Technology 15: $128-136$.

Moscoso C, Vélez M, Flores A, Agudelo N (1995) Effects of guanacaste tree (Enterolobium cyclocarpum Jacq. Griseb.) fruit as replacement for sorghum grain and cotton-seed meal in lamb diets. Small Ruminant Research 18: 121-124.

$\mathrm{Na}$ Y, Li DH, Lee SR (2017) Effects of dietary forage-to-concentrate ratio on nutrient digestibility and enteric methane production in growing goats (Capra hircus) and Sika deer (Cervus Nippon hortulorum). AsianAustralasian Journal of Animal Sciences 30: 967-972.

NRC (1985) Nutrient requirements of sheep. Sixth Edition. National Academy Press. Washington DC, USA. 99p.

Oba M, Allen MS (1999) Evaluation of the importance of the digestibility of neutral detergent fiber from forage: effects on dry matter intake and milk yield of dairy cows. Journal of Dairy Science 82: 589-596.

Olivares-Pérez J, Avilés-Nova F, Albarran-Portillo B, Castelán-Ortega OA, Rojas-Hernández S (2013) Use of three fodder trees in the feeding of goats in the subhumid tropics in Mexico. Tropical Animal and Production 45: 821-828.

Ørskov ER, Hovell FD, Mould F (1980) The use of the nylon bag technique for the evaluation of feedstuffs. Tropical Animal Production 5: 195-213.

Peralta N, Palma JM, Macedo R (2004) Efecto de diferentes niveles de inclusión de parota (Enterolobium cyclocarpum) en el desarrollo de ovinos en estabulación. Livestock Research for Rural Development 16: 1.

Pinto R, Gómez H, Martínez B, Hernández A, Medina F, Ortega L, et al. (2004) Especies forrajeras utilizadas bajo silvo-pastoreo en el centro de Chiapas. Avances en Investigación Agropecuaria 8: 53-67.

Piñeiro AT, Ayala BAJ, Chay CAJ, Ku VJC (2013) Dry matter intake and digestibility of rations replacing concentrates with graded levels of Enterolobium cyclocarpum in Pelibuey lambs. Tropical Animal Health and Production 45: 577-583.

Rojas HS, Olivares P, Aviles NJF, Villa MA, Reynoso PA, Camacho DLM (2015) Productive response of lambs fed Crescentia alata and Guazuma ulmifolia fruits in a tropical region of Mexico. Tropical Animal Health and Production 47: 1431-1436.

Román MML, Palma JM, Zorrilla J, Mora A, Gallegos A (2008) Degradabilidad in situ de la materia seca de la harina del fruto de guásima, Guazuma ulmifolia, con dietas de frutos de especies arbóreas. Zootecnia Tropical 26: 227-230.

Salisbury MW, Krehbiel CR, Ross TT, Schultz CL, Melton LL (2004) Effects of supplemental protein type on intake, nitrogen balance, and site, and extent of digestion in whiteface wethers consuming low-quality grass hay. Journal of Animal Science 82: 3567-3576.

SAS (2004) SAS/STAT users guide. Versión 9.1. SAS Institute Inc. Cary NC, U.S.A. 5121p.

Schneider BH, Flatt WP (1975) The evaluation of feeds through digestibility experiments. University of Georgia Press. 423p.

Sosa D, Larco C, Falconí R, Toledo D, Suárez G (2006) Digestibilidad de maralfalfa (Pennisetum sp.) en cabras. Boletín Técnico 5, Serie Zoológica 2: 68-76.

Tilley JM, Terry RA (1963) A two-stage technique for the in vitro digestion of forage crops. Journal of the British Grassland Society 28: 104-111. 
Van Soest PJ (1994) Nutritional Ecology of the Ruminant. 2nd Edition. Cornell University Press. New York, USA. 476p.

Xu J, Hou V, Yang H, Shi R, Wu C, Huo Y, Zhao G (2014) Effects of forage sources on rumen fermentation characteristics, performance, and microbial protein synthesis on midlactation cows. Asian-Australasian Journal of Animal Sciences 27: 667-673.

Zabala R (1995) Producción de forraje bajo corte de cultivares de alfalfa sin reposo invernal en condiciones de riego en el valle inferior del Río Negro. Revista Argentina de Producción Animal 15: 266-268.

Zardo RN, Henriques RPB (2011) Growth and fruit production of the tree Caryocar brasiliense in the Cerrado of central Brazil. Agroforestry Systems 82: 15-23. 